\title{
Positive solutions for a nonlinear elliptic problem with strong lack of compactness*
}

\author{
Riccardo Molle \\ Dipartimento di Matematica \\ Università di Roma "Tor Vergata" \\ Via della Ricerca Scientifica $n .1$ \\ 00133 Roma, Italy \\ E-mail: molle@mat.uniroma2.it
}

\begin{abstract}
This paper deals with the lack of compactness in nonlinear elliptic problems $(P)$. In particular, a domain $\Omega$ is provided where not converging Palais-Smale sequences exist at every energy level. Nevertheless, it is proved that problem $(P)$ has infinitely many solutions on $\Omega$.
\end{abstract}

Key words: Lack of compactness; Nonlinear elliptic problems; Domains with unbounded boundary.

A.M.S. subject classification 2000: 35J20; 35J65.

\section{Introduction, examples and statement of the re- sult}

In this paper we are concerned with the failure of compactness related to problem

$$
(P) \quad \begin{cases}-\Delta u+u=|u|^{p-2} u & \text { in } \Omega, \\ u>0 & \text { in } \Omega, \\ u \in H_{0}^{1}(\Omega) & \end{cases}
$$

when $\Omega \subset \mathbb{R}^{N}, N \geq 3$, is an unbounded domain, having unbounded boundary, and $2<p<2^{*}=2 N /(N-2)$.

${ }^{*}$ Work supported by the Italian national research project "Metodi variazionali e topologici nello studio di fenomeni non lineari". 
There are many studies related to problem $(P)$, in recent years, because of its connection with questions arising from applied sciences. A typical approach is the variational one: the solutions of Problem $(P)$ can be found in correspondence to positive functions that are critical points of the "energy" functional $\mathcal{E}: M \rightarrow \mathbb{R}$ given by

$$
\mathcal{E}(u)=\int_{\Omega}\left(|\nabla u|^{2}+u^{2}\right) d x
$$

where $M$ is the manifold

$$
M=\left\{u \in H_{0}^{1}(\Omega):|u|_{L^{p}(\Omega)}=1\right\} .
$$

When $\Omega$ is bounded, classical variational methods can be applied, taking advantage of the compactness of the embedding $H_{0}^{1}(\Omega) \hookrightarrow L^{p}(\Omega)$, so problem $(P)$ always has a solution, corresponding to $\min _{M} \mathcal{E}$, and the multiplicity of solutions is related to the shape of $\Omega$ (see, for example, [4, 14] and references therein).

The key compactness condition is the Palais-Smale condition: $\mathcal{E}$ satisfies the Palais-Smale condition if every sequence $\left(u_{n}\right)_{n}$ in $M$ such that $\lim _{n \rightarrow+\infty} \mathcal{E}\left(u_{n}\right)=c, c \in \mathbb{R}$, $\lim _{n \rightarrow+\infty} \nabla \mathcal{E}\left(u_{n}\right)=0$ (a Palais Smale sequence), has a converging subsequence.

If $\Omega$ is unbounded the situation is different from the bounded case. Of course, when the embedding $H_{0}^{1}(\Omega) \hookrightarrow L^{p}(\Omega)$ is compact, it is again possible to apply the variational techniques used in the bounded case. This happens, for example, if $\Omega$ is "thin" at infinity, in the sense that

$$
\lim _{R \rightarrow+\infty} \sup \left\{\mu(B(x, 1) \cap \Omega): x \in \mathbb{R}^{N},|x|=R\right\}=0,
$$

where $\mu$ denotes the Lebesgue measure and $B(x, 1)$ is the unitary ball with centre in $x$. So, let us consider the case when $\Omega$ is not thin at infinity. If $\Omega=\mathbb{R}^{N},(P)$ has a solution $\omega$, unique up to translations, corresponding to

$$
m:=\min \left\{\|u\|_{H^{1}\left(\mathbb{R}^{N}\right)}^{2}: u \in H^{1}\left(\mathbb{R}^{N}\right),|u|_{L^{p}\left(\mathbb{R}^{N}\right)}=1\right\} .
$$

When $\Omega$ looks like $\mathbb{R}^{N}$ at infinity, in the sense that $\mathbb{R}^{N} \backslash \Omega$ is bounded (i.e. $\Omega$ is an exterior domain), then the analysis of the compactness failure shows that a PalaisSmale sequence of $\mathcal{E}$ differs from its weak limit by "waves" $\omega$ that go to infinity. This behaviour of the Palais-Smale sequences ensures that a local compactness condition holds. Taking into account this property, existence and multiplicity results for problem $(P)$ in exterior domains are proved (see [3, 2, 11] and references therein).

When not only $\Omega$, but also $\mathbb{R}^{N} \backslash \Omega$ is unbounded, the existence of solutions of $(P)$ depends on the shape of $\Omega$. For example in [8] it is proved that if there exists $\bar{x} \in \mathbb{R}^{N}$ such that $(\nu(x), \bar{x}) \geq 0 \forall x \in \partial \Omega,(\nu(x), \bar{x}) \not \equiv 0$, then problem $(P)$ has no solution, while in [7] it is shown that solutions for $(P)$ exist in "strip-like" domains (see also 
the monograph [13], and references therein, for other existence results in domains with unbounded boundary).

In [10] a general condition on the shape of $\Omega$ at infinity is stated such that, even if both $\Omega$ and $\mathbb{R}^{N} \backslash \Omega$ are unbounded, a local compactness condition still holds. The condition stated in [10] requires that $\Omega$ enlarges, at infinity, and its boundary flattens, or shrinks, at infinity. Namely, the following conditions are required:

$$
\left\{\begin{array}{l}
\lim _{R \rightarrow+\infty} \inf \{r(x): x \in \Omega,|x|=R\}=+\infty \\
\lim _{R \rightarrow+\infty} \sup \{h(y): y \in \partial \Omega,|y|=R\}=0
\end{array}\right.
$$

where

$$
\begin{gathered}
r(x)=\sup \{\rho>0: \exists \bar{x} \in \Omega \text { such that } x \in B(\bar{x}, \rho) \text { and } B(\bar{x}, \rho) \subset \Omega\}, \\
h(y)=\sup \left\{\operatorname{dist}\left(z, T_{\partial \Omega, y} \cap B(y, 1)\right): z\right. \text { is in the connected component } \\
\text { of } \partial \Omega \cap B(y, 1) \text { containing } y\}
\end{gathered}
$$

(here $T_{\partial \Omega, y}$ is the hyperplane tangent to $\partial \Omega$ in $y$ ). It is worth to remark that conditions $\left(C_{1}\right)$ and $\left(C_{2}\right)$, hence local compactness, is not sufficient to guarantee the existence of solutions of $(P)$; for example, an half-space $\Pi$ verifies $\left(C_{1}\right)$ and $\left(C_{2}\right)$, but $(P)$ has no solution on $\Pi$, by the Esteban-Lions result. What happens in half-spaces is that, actually, no Palais-Smale sequences for $\mathcal{E}$ exist at the compactness levels.

Domains $\Omega$ with unbounded boundary and periodic in some directions (for example the exterior of a cylinder) are considered in [6] and a result of multiplicity of solutions for $(P)$ is proved. Let us remark that every solution $\bar{u}$, found in [6], provides a noncompact family of solutions of $(P)$ (hence a non-compact Palais-Smale sequence at the level $\mathcal{E}\left(\frac{\bar{u}}{|\bar{u}|_{L^{p}(\Omega)}}\right)$ ) by moving $\bar{u}$ according to the periodic structure of $\Omega$.

Now, one can look for a domain $\Omega$ with no periodicity assumption and such that not converging Palais-Smale sequences for $\mathcal{E}$ exist at every energy level. It is worth to observe that in a domain with this property no local compactness condition can hold.

Looking in finite dimension, a function $f \in \mathcal{C}^{1}([0,+\infty),(0,+\infty))$ such that

$$
f(i)=q_{i}, \quad f^{\prime}(i)=0 \quad \forall i \in \mathbb{N}, \quad \text { where }\left(q_{i}\right)_{i}=\mathbb{Q}^{+},
$$

has such a strong failure of compactness. Indeed for every $s \in[0,+\infty)$ we can consider $\left(q_{i_{j}}\right)_{j}$ in $\mathbb{Q}^{+}$such that $\lim _{j \rightarrow+\infty} f\left(i_{j}\right)=q_{i_{j}}=s$ and so, taking into account (1.2), we have that $\left(i_{j}\right)$ is a not converging Palais-Smale sequence for $f$ at the level $s$. Observe that every $q \in \mathbb{Q}$ is a critical level for $f$, i.e. the critical levels are a dense subset of the range $(0,+\infty)$ (no more can be required by the Sard's Theorem). It is also possible to consider a function $g \in \mathcal{C}^{1}\left(\mathbb{R}^{2},(0,+\infty)\right)$ such that

$$
g(i, 0)=q_{i}, \quad \nabla g(i, 0)=\left(\frac{1}{i}, \frac{1}{i}\right), \quad \frac{\partial g}{\partial y}(x, y)>0 \quad \forall(x, y) \in \mathbb{R}^{2} .
$$


In this second example we see that there is the same strong failure of the compactness condition of the previous example and $g$ has no critical point.

Going back to the functional $\mathcal{E}$, define

$$
S_{i}=\left\{\left(x_{1}, \ldots, x_{N}\right) \in \mathbb{R}^{N}:-\frac{1}{2} q_{i}<x_{N}<\frac{1}{2} q_{i}\right\},
$$

$\left(\left(q_{i}\right)_{i}=\mathbb{Q}^{+}\right)$and set

$$
\begin{aligned}
\Omega= & \left\{x=\left(x_{1}, \ldots, x_{N}\right) \in \mathbb{R}^{N}: \sum_{j=1}^{N-1} x_{j}^{2}<1\right\} \cup \\
& S_{1} \cup\left[\bigcup_{i=2}^{\infty}\left[S_{i}+\left(0, \ldots, 0, i+\sum_{j=1}^{i-1} q_{j}+\frac{1}{2} q_{i}\right)\right]\right] .
\end{aligned}
$$

The domain $\Omega$ is introduced in [9], where it is seen that the following properties hold.

Proposition 1.1 It holds:

a) $\{\mathcal{E}(u): u \in M\}=(m,+\infty)$;

b) for every $c \in[m,+\infty)$ there exists a Palais-Smale sequence $\left(u_{n}\right)_{n}$ for $\mathcal{E}$ at the level c, i.e.

$$
\lim _{n \rightarrow+\infty} \mathcal{E}\left(u_{n}\right)=c, \quad \lim _{n \rightarrow+\infty} \nabla \mathcal{E}\left(u_{n}\right)=0,
$$

that is not relatively compact.

For the reader's convenience, a proof of Proposition 1.1 is contained in Section 2. Here we stress the fact that not converging Palais-Smale sequence for $\mathcal{E}$ are related either to translations in the $x_{i}$-directions, for $i=1, \ldots, N-1$, either to translations in the $x_{N}$-direction.

In this paper we prove that, in spite of the strong failure in compactness described above and of the non periodic structure of $\Omega$, problem $(P)$ on $\Omega$ has infinitely many solutions.

Theorem 1.2 Problem $(P)$ has a sequence of solutions $\left(v_{n}\right)_{n}$ such that

$$
\lim _{n \rightarrow+\infty} \mathcal{E}\left(\frac{v_{n}}{\left|v_{n}\right|_{L^{p}(\Omega)}}\right)=m
$$

The solutions given by Theorem 1.2 correspond to local minima, that are "localized" in large strips. We reach the local minima by following maximal slope curves. In order to construct such curves, a basic tool is the analysis of the Palais-Smale sequences (§3). Indeed, we show that Palais-Smale sequences are compact on a maximal slope curve, near the local minima.

Finally, let us remark that if we consider the domain

$$
\mathcal{D}=\Omega \cup\left\{x=\left(x_{1}, \ldots, x_{N}\right) \in \mathbb{R}^{N}: x_{1}<0\right\}
$$


then Proposition 1.1 still holds, on $\mathcal{D}$, but problem $(P)$ has no solution, because $\mathcal{D}$ satisfies the Esteban-Lions condition.

The paper is organized as follows: Section 2 contains the proof of Proposition 1.1, the variational setting and some useful tools; Section 3 is devoted to the analysis of the Palais-Smale sequences and in Section 4 Theorem 1.2 is proved.

\section{Preliminary results, the variational setting and useful tools}

In this paper the following notations are used:

- if $\mathcal{D} \subseteq \mathbb{R}^{N}$ and $u \in H_{0}^{1}(\mathcal{D})$, we denote also by $u$ its extension to $\mathbb{R}^{N}$ obtained by setting $u \equiv 0$ outside $\mathcal{D}$.

- $L^{p}(\mathcal{D}), 1 \leq p<+\infty, \mathcal{D} \subseteq \mathbb{R}^{N}$, denotes the usual Lebesgue space, endowed with the norm $|u|_{L^{p}(\mathcal{D})}=\left(\int_{\mathcal{D}}|u|^{p}\right)^{\frac{1}{p}} ;$ if $\mathcal{D}=\mathbb{R}^{N}$, we simply write $|u|_{L^{p}\left(\mathbb{R}^{N}\right)}=|u|_{p}$

- $H_{0}^{1}(\mathcal{D}), \mathcal{D} \subseteq \mathbb{R}^{N}$ denotes the Sobolev space obtained as the closure of $\mathcal{C}_{0}^{\infty}(\mathcal{D})$ with respect to the norm

$$
\|u\|_{H^{1}(\mathcal{D})}=\left[\int_{\mathcal{D}}\left(|\nabla u|^{2}+u^{2}\right) d x\right]^{\frac{1}{2}}
$$

if $\mathcal{D}=\mathbb{R}^{N}$, we simply write $\|u\|_{H^{1}\left(\mathbb{R}^{N}\right)}=\|u\|$.

- The generic point $x=\left(x_{1}, \ldots, x_{N-1}, x_{N}\right) \in \mathbb{R}^{N}$ is denoted by $\left(x^{\prime}, x_{N}\right)$, where $x^{\prime}=$ $\left(x_{1}, \ldots, x_{N-1}\right) \in \mathbb{R}^{N-1}$ and $x_{N} \in \mathbb{R} ;$ we put also $\left|x^{\prime}\right|=\left(\sum_{j=1}^{N-1} x_{j}^{2}\right)^{\frac{1}{2}}$.

- We set the points $Q_{1}^{-}=\left(0, \ldots, 0,-\frac{q_{1}}{2}\right), Q_{1}^{+}=\left(0, \ldots, 0, \frac{q_{1}}{2}\right)$ and, for $i=2,3, \ldots$, $Q_{i}^{-}=\left(0, \ldots, 0, i+\sum_{j=1}^{i-1} q_{j}\right), \quad Q_{i}^{+}=\left(0, \ldots, 0, i+\sum_{j=1}^{i} q_{j}\right)$.

Proof of Proposition 1.1. First, we prove that

$$
\inf _{M} \mathcal{E}=m
$$

Let us recall that $m$ in (1.1) is achieved by a positive function $\omega$, radially symmetric and decreasing when the radial co-ordinate increases, that is unique modulo translations and satisfies

$$
\begin{gathered}
\lim _{|x| \rightarrow+\infty}|\omega(x)||x|^{\frac{N-1}{2}} e^{|x|}=d>0, \\
\lim _{|x| \rightarrow+\infty}|\nabla \omega(x)||x|^{\frac{N-1}{2}} e^{|x|}=d,
\end{gathered}
$$


for a suitable positive constant $d$ (see [2] and references therein).

For $R>0$, let $\varphi_{R}: \mathbb{R}^{N} \rightarrow \mathbb{R}$ be a cut-off function defined by $\varphi_{R}(x)=\varphi\left(\frac{|x|}{R}\right)$, where $\varphi \in \mathcal{C}^{\infty}\left(\mathbb{R}^{+},[0,1]\right)$ is a non-increasing function such that $\varphi(t)=1$ if $t \in\left[0, \frac{1}{4}\right]$, $\varphi(t)=0$ if $t \in\left[\frac{1}{2},+\infty\right)$, and define

$$
u_{i}(x)=\frac{\varphi_{q_{i}}\left(x-\frac{Q_{i}^{-}+Q_{i}^{+}}{2}\right) \omega\left(x-\frac{Q_{i}^{-}+Q_{i}^{+}}{2}\right)}{\left|\varphi_{q_{i}}\left(x-\frac{Q_{i}^{-}+Q_{i}^{+}}{2}\right) \omega\left(x-\frac{Q_{i}^{-}+Q_{i}^{+}}{2}\right)\right|_{p}} .
$$

It is clear that $u_{i} \in M, \forall i \in \mathbb{N}$, and, taking into account (2.2) and (2.3), we have

$$
\begin{aligned}
& \lim _{q_{i} \rightarrow+\infty}\left|\varphi_{q_{i}}\left(x-\frac{Q_{i}^{-}+Q_{i}^{+}}{2}\right) \omega\left(x-\frac{Q_{i}^{-}+Q_{i}^{+}}{2}\right)-\omega\left(x-\frac{Q_{i}^{-}+Q_{i}^{+}}{2}\right)\right|_{p}=0, \\
& \lim _{q_{i} \rightarrow+\infty}\left\|\varphi_{q_{i}}\left(x-\frac{Q_{i}^{-}+Q_{i}^{+}}{2}\right) \omega\left(x-\frac{Q_{i}^{-}+Q_{i}^{+}}{2}\right)-\omega\left(x-\frac{Q_{i}^{-}+Q_{i}^{+}}{2}\right)\right\|=0 .
\end{aligned}
$$

From (2.4) and (2.5) we infer $\lim _{q_{i} \rightarrow+\infty}\left\|u_{i}(x)-\omega\left(x-\frac{Q_{i}^{-}+Q_{i}^{+}}{2}\right)\right\|=0$, i.e.

$$
\lim _{q_{i} \rightarrow+\infty} \mathcal{E}\left(u_{i}\right)=m
$$

that implies $\inf _{M} \mathcal{E} \leq m$. On the other hand $\inf _{M} \mathcal{E} \geq m$ directly follows from (1.1), so (2.1) is proved.

We claim that the infimum in (2.1) is not achieved. Assume, by contradiction, that a minimum point $u^{*} \in M$ exists. By the uniqueness of the minimizers of (1.1), there exists $y^{*} \in \mathbb{R}^{N}$ such that $u^{*}(x)=\omega\left(x-y^{*}\right), \forall x \in \mathbb{R}^{N}$. This is not possible because $u^{*} \equiv 0$ on $\mathbb{R}^{N} \backslash \Omega$, while $\omega>0$ on $\mathbb{R}^{N}$, so we have proved the claim.

Recall, now, that for every $i \in \mathbb{N}$ there exists a critical point $z_{i}$ for $\mathcal{E}$ on $\{u \in$ $\left.H_{0}^{1}\left(S_{i}\right):|u|_{p}=1\right\}\left(S_{i}\right.$ is introduced in (1.4) $)$, corresponding to the "minimal" solution of the problem $(P)$ on $S_{i}$ (see [7]). Namely, $z_{i}$ satisfies

$$
\Theta\left(q_{i}\right):=\left\|z_{i}\right\|^{2}=\min \left\{\|u\|^{2}: u \in H_{0}^{1}\left(S_{i}\right),|u|_{p}=1\right\} .
$$

It is easily seen that the above introduced $\Theta: \mathbb{Q}^{+} \rightarrow \mathbb{R}$ is a continuous monotone decreasing function and we claim that

$$
\begin{gathered}
\lim _{q_{i} \rightarrow+\infty} \Theta\left(q_{i}\right)=m, \\
\lim _{q_{i} \rightarrow 0} \Theta\left(q_{i}\right)=+\infty .
\end{gathered}
$$

Taking into account (2.7), in order to show (2.8) we can use the test functions $\frac{\varphi_{q_{q_{i}}} \omega}{\left|\varphi_{q_{i}} \omega\right|_{p}}$ and proceed with the same argument developed to prove (2.1). 
To prove (2.9) observe that, by the Poincaré's inequality on a strip, there exists a constant $\bar{k}>0$ such that

$$
|u|_{p} \leq \bar{k}|\nabla u|_{L^{2}\left(S_{1}\right)} \quad \forall u \in H_{0}^{1}\left(S_{1}\right)
$$

(see Theorem 6.30 in [1], for example). To simplify the notation, let us assume $q_{1}=1$ and set $\hat{z}_{i}(x)=z_{i}\left(q_{i} x\right)$; applying (2.10) to $\hat{z}_{i}$ and taking into account that $\left|z_{i}\right|_{p}=1$ $\forall i \in \mathbb{N}$, we get

$$
q_{i}^{-\frac{(N-2)}{2 p}\left(\frac{2 N}{N-2}-p\right)} \leq \bar{k}\left|\nabla z_{i}\right|_{L^{2}\left(S_{i}\right)}<\bar{k}\left\|z_{i}\right\|
$$

that implies (2.9).

Now we are in position to verify $(b)$. If $c=\Theta\left(q_{i}\right)$, for some $i \in \mathbb{N}$, let us construct the sequence $\left(z_{i, j}\right)_{j}$ by

$$
z_{i, j}\left(x_{1}, \ldots, x_{N}\right)=z_{i}\left(x_{1}+j, x_{2}, \ldots, x_{N-1}, x_{N}-\frac{Q_{i}^{-}+Q_{i}^{+}}{2}\right) .
$$

Then, it is not difficult to see that $\left(z_{i, j}\right)_{j}$ is a Palais-Smale sequence for $\mathcal{E}$ at the level $c$, and it cannot have converging subsequence because it weakly converges to zero.

If $c \in[m,+\infty) \backslash \Theta\left(\mathbb{Q}^{+}\right)$, let $\left(q_{i_{j}}\right)_{j}$ be a sequence in $\mathbb{Q}$ such that $c=\lim _{j \rightarrow+\infty} \Theta\left(q_{i_{j}}\right)$. Then $\left(z_{i_{j}, j}\right)_{j}$ is a Palais-Smale sequence for $\mathcal{E}$ at the level $c$ that has no converging subsequences.

q.e.d.

From the minimality of $m$ (see (1.1) ) and $(a)$ of Proposition 1.1 we get the following results, which we need in the sequel.

Proposition 2.1 Let $\mu>0$ and $\mathcal{D} \subseteq \mathbb{R}^{N}$ be an open domain with piecewise smooth boundary (if not empty). If $\bar{u}$ is a nontrivial solution of

$$
P(\mu, \mathcal{D}) \quad\left\{\begin{array}{l}
-\Delta u+u=\mu|u|^{p-2} u \quad \text { in } \mathcal{D}, \\
u \in H_{0}^{1}(\mathcal{D})
\end{array}\right.
$$

then

$$
|\bar{u}|_{p}>\left(\frac{m}{\mu}\right)^{\frac{1}{p-2}} \quad \text { if } \mathcal{D} \neq \mathbb{R}^{N}, \quad|\bar{u}|_{p} \geq\left(\frac{m}{\mu}\right)^{\frac{1}{p-2}} \quad \text { if } \mathcal{D}=\mathbb{R}^{N} .
$$

Proof By (1.1),

$$
m|\bar{u}|_{p}^{2} \leq\|\bar{u}\|^{2}
$$

and, as we have seen in the proof of Proposition 1.1, the equality in (2.13) can hold only if $\mathcal{D}=\mathbb{R}^{N}$. Moreover, $\bar{u}$ being a solution of $P(\mu, \mathcal{D})$,

$$
\|\bar{u}\|^{2}=\mu|\bar{u}|_{p}^{p}
$$


Thus

$$
|\bar{u}|_{p}^{p-2} \geq \frac{m}{\mu}
$$

where equality holds only if $\mathcal{D}=\mathbb{R}^{N}$, so (2.12) follows.

q.e.d.

Corollary 2.2 Let $\bar{u}$ be a critical point of $\mathcal{E}$. If $\mathcal{E}(\bar{u}) \in\left(m, 2^{1-\frac{2}{p}} m\right]$ then $\bar{u}$ does not change sign.

Proof Let $\bar{u}=\bar{u}^{+}-\bar{u}^{-}$, with $\bar{u}^{+} \not \equiv 0$ and $\bar{u}^{-} \not \equiv 0$, and call $\mathcal{E}(\bar{u})=\mu$. Then, by $(a)$ in Proposition 1.1 and since $\bar{u}$ solves $P(\mu, \Omega)$, we have

$$
m\left|\bar{u}^{ \pm}\right|_{p}^{2}<\left\|\bar{u}^{ \pm}\right\|^{2}=\mu\left|\bar{u}^{ \pm}\right|_{p}^{p} .
$$

From (2.14) and $|\bar{u}|_{p}=1$ we infer

$$
1=|\bar{u}|_{p}^{p}=\left|\bar{u}^{+}\right|_{p}^{p}+\left|\bar{u}^{-}\right|_{p}^{p}>2\left(\frac{m}{\mu}\right)^{\frac{p}{p-2}},
$$

that implies

$$
\mu>2^{1-\frac{2}{p}} m
$$

q.e.d.

In view of the axial symmetry of the problem and taking into account the principle of symmetric criticality (see [12], or also [15], Theorem 1.28), to solve problem $(P)$ it is sufficient to consider the subspace of the axially symmetric functions

$$
H_{0, r}^{1}(\Omega)=\left\{u \in H_{0}^{1}(\Omega): u\left(x^{\prime}, x_{N}\right)=u\left(\bar{x}^{\prime}, x_{N}\right) \text { if }\left|x^{\prime}\right|=\left|\bar{x}^{\prime}\right|\right\},
$$

the smooth manifold

$$
V=\left\{u \in H_{0, r}^{1}(\Omega):|u|_{p}=1\right\}
$$

and look for critical points of the functional $E=\mathcal{E}_{\mid V}$.

Remark 2.3 Let us observe that we cannot find critical points of $E$ simply by minimization. Indeed, the argument developed in the proof of $(a)$ of Proposition 1.1 shows that

$$
\inf _{V} E=m
$$

and that the infimum in (2.15) is not achieved. 
Now, we denote the $x_{N}$-axis by

$$
A=\left\{x=\left(x_{1}, \ldots, x_{N}\right) \in \mathbb{R}^{N}: x_{1}=\ldots=x_{N-1}=0\right\} .
$$

Then, we introduce a barycenter type function on $H_{0, r}^{1}\left(\mathbb{R}^{N}\right) \backslash\{0\}$ : for $u \in H_{0, r}^{1}\left(\mathbb{R}^{N}\right) \backslash\{0\}$ set

$$
\begin{array}{cc}
\tilde{u}(x)=\frac{1}{\mu(B(x, 1))} \int_{B(x, 1)}|u(y)| d y & \forall x \in \mathbb{R}^{N}, \\
\hat{u}(x)=\left[\tilde{u}(x)-\frac{1}{2} \max _{\mathbb{R}^{N}} \tilde{u}(x)\right]^{+} \quad \forall x \in \mathbb{R}^{N}
\end{array}
$$

and define $\beta: H_{0, r}^{1}\left(\mathbb{R}^{N}\right) \backslash\{0\} \rightarrow A$ by

$$
\beta(u)=\frac{1}{|\hat{u}|_{p}^{p}} \int_{\mathbb{R}^{N}}(\hat{u}(x))^{p} x d x .
$$

Let us remark that $\beta$ is well defined for all $u \in H_{0, r}^{1}\left(\mathbb{R}^{N}\right) \backslash\{0\}$, because $\hat{u} \not \equiv 0$ and has compact support, $\beta(u) \in A$ because $u$ is axially symmetric and, moreover, $\beta$ is continuous and verifies:

$$
\begin{gathered}
\beta(u(x-y))=\beta(u(x))+y \quad \forall u \in H_{0, r}^{1}\left(\mathbb{R}^{N}\right) \backslash\{0\}, \forall y \in A, \\
\beta(\omega(x))=0 .
\end{gathered}
$$

\section{Behaviour of Palais-Smale sequences of $E$}

In the next Proposition we study Palais-Smale sequences in the energy range $[m$, $2^{1-\frac{2}{p}} m$ ) (see Remark 3.2 for the level $2^{1-\frac{2}{p}} m$ ). We show that or such a sequence is relatively compact or it is composed by a unique "wave" that goes to infinity, in the $x_{N}$-direction, and converges to a limit problem.

Proposition 3.1 Let $\left(u_{n}\right)_{n}$ be a Palais-Smale sequence for $E$ at level $c$. If $c<2^{1-\frac{2}{p}} m$ then, up to a subsequence, one of the following alternatives is possible:

a) $\left(u_{n}\right)_{n}$ converges to a function $u_{0}$ in $H_{0, r}^{1}(\Omega)$;

b) $\left|\beta\left(u_{n}\right)\right| \longrightarrow+\infty$.

Proof Since $\left(u_{n}\right)_{n}$ is a Palais-Smale sequence for $E$, in particular it is bounded in $H_{0, r}^{1}(\Omega)$, so there exists $u_{0} \in H_{0, r}^{1}(\Omega)$ such that, up to a subsequence,

$$
u_{n} \longrightarrow u_{0} \quad \text { weakly in } H_{0}^{1}(\Omega) \text { and in } L^{p}(\Omega) \text {, a.e. in } \Omega \text { and in } L_{\text {loc }}^{p}\left(\mathbb{R}^{N}\right) .
$$


Furthermore, there exists a sequence $\left(\mu_{n}\right)_{n}$ in $\mathbb{R}$ such that

$$
\left(\nabla E\left(u_{n}\right), w\right)=\int_{\Omega}\left(\nabla u_{n} \cdot \nabla w+u_{n} w\right)=\mu_{n} \int_{\Omega}\left|u_{n}\right|^{p-2} u_{n} w+o(1)\|w\| \quad \forall w \in H_{0, r}^{1}(\Omega) .
$$

Setting $w=u_{n}$ in (3.2), we get

$$
\lim _{n \rightarrow+\infty} \mu_{n}=c
$$

So, $u_{0}$ verifies

$$
\int_{\Omega}\left(\nabla u_{0} \cdot \nabla w+u_{0} w\right)=c \int_{\Omega}\left|u_{0}\right|^{p-2} u_{0} w \quad \forall w \in H_{0, r}^{1}(\Omega)
$$

and, taking into account the principle of symmetric criticality, it is a solution of

$$
\left\{\begin{array}{l}
-\Delta u+u=c|u|^{p-2} u \quad \text { in } \Omega \\
u \in H_{0}^{1}(\Omega)
\end{array}\right.
$$

Now, set

$$
v_{n}(x)=u_{n}(x)-u_{0}(x) .
$$

If $v_{n} \rightarrow 0$ in $H_{0}^{1}(\Omega)$, we are done, taking into account the continuity of $\beta$, otherwise there exists a constant $k_{0}>0$ such that, up to a subsequence,

$$
\left\|v_{n}\right\| \geq k_{0}>0 \quad \forall n \in \mathbb{N}
$$

We are proving that in such a case $u_{0} \equiv 0$ and $(b)$ holds.

The sequence $\left(v_{n}\right)_{n}$ is uniformly bounded in $H_{0, r}^{1}(\Omega)$ and, by (3.1),

$$
v_{n} \longrightarrow 0 \quad \text { weakly in } H_{0}^{1}(\Omega) \text { and in } L^{p}(\Omega) \text {, a.e. in } \Omega \text { and in } L_{\text {loc }}^{p}\left(\mathbb{R}^{N}\right) \text {; }
$$

moreover, a direct computation shows that

$$
\left\|v_{n}\right\|^{2}=\left\|u_{n}\right\|^{2}-\left\|u_{0}\right\|^{2}+o(1)
$$

and, by the Brezis-Lieb Lemma ([5]),

$$
\left|v_{n}\right|_{p}^{p}=\left|u_{n}\right|_{p}^{p}-\left|u_{0}\right|_{p}^{p}+o(1) .
$$

Observe that, by (3.2), (3.3), (3.4) and (3.9),

$$
\left\|v_{n}\right\|^{2}=c\left(\left|u_{n}\right|_{p}^{p}-\left|u_{0}\right|_{p}^{p}\right)+o(1)
$$

hence, from (3.10), $\left|v_{n}\right|_{p}^{p} \geq k_{1}>0$ follows, for a suitable constant $k_{1}>0$. 
Now, let us decompose $\mathbb{R}^{N}$ into the $N$-dimensional hypercubes $Q_{l}$, having unitary sides and vertices with integer co-ordinates and put, for all $n \in \mathbb{N}$,

$$
d_{n}=\max _{l \in \mathbb{N}}\left|v_{n}\right|_{L^{p}\left(Q_{l}\right)}
$$

We claim that there exists $\gamma>0$ such that

$$
d_{n} \geq \gamma>0 \quad \forall n \in \mathbb{N}
$$

indeed

$$
\begin{aligned}
0<k_{1} & \leq\left|v_{n}\right|_{p}^{p}=\sum_{l \in \mathbb{N}}\left|v_{n}\right|_{L^{p}\left(Q_{l}\right)}^{p} \leq \max _{l \in \mathbb{N}}\left|v_{n}\right|_{L^{p}\left(Q_{l}\right)}^{p-2} \sum_{l \in \mathbb{N}}\left|v_{n}\right|_{L^{p}\left(Q_{l}\right)}^{2} \\
& \leq d_{n}^{p-2} s \sum_{l \in \mathbb{N}}\left\|v_{n}\right\|_{H^{1}\left(Q_{l}\right)}^{2}=d_{n}^{p-2} s\left\|v_{n}\right\|^{2}
\end{aligned}
$$

where $s$ is given by the embedding $H^{1}\left(Q_{l}\right) \hookrightarrow L^{p}\left(Q_{l}\right)$ (it is indipendent of $l$ ).

For all $n \in \mathbb{N}$, let $y_{n}=\left(y_{n}^{\prime}, y_{n, N}\right)$ be the center of an hypercube $Q_{n}$ where

$$
\left|v_{n}\right|_{L^{p}\left(Q_{n}\right)} \geq \gamma
$$

Since the functions $\left(v_{n}\right)_{n}$ are radially symmetric with respect to the $x_{N}$-axis and uniformly bounded in $L^{p}\left(\mathbb{R}^{N}\right)$, by using (3.15) we get

$$
\left|y_{n}^{\prime}\right| \leq k
$$

for a suitable constant $k>0$. Hence we must have

$$
\left|y_{n, N}\right| \longrightarrow+\infty
$$

because $v_{n} \rightarrow 0$ in $L_{\mathrm{loc}}^{p}\left(\mathbb{R}^{N}\right)$.

Now, let us set $\tilde{y}_{n}=\left(0, \ldots, 0, y_{n, N}\right)$ and call $\tilde{v}_{0}$ the weak limit, in $H^{1}\left(\mathbb{R}^{N}\right)$ and in $L^{p}\left(\mathbb{R}^{N}\right)$, of $\tilde{v}_{n}(x)=v_{n}\left(x+\tilde{y}_{n}\right)$, up to a subsequence. From (3.15), (3.16) and Rellich Theorem we infer $\tilde{v}_{0} \not \equiv 0$ and, taking into account (3.2) and (3.3), $\tilde{v}_{0}$ solves problem $P(c, \mathcal{D})$, on its domain $\mathcal{D}$ (see(2.11) $)$.

So, it is possible to conclude that $u_{0} \equiv 0$. Indeed (3.9) implies $\left\|u_{n}\right\|^{2} \geq\left\|u_{0}\right\|^{2}+$ $\left\|\tilde{v}_{0}\right\|^{2}+o(1)$, thus, if $u_{0}$ were not zero, by Proposition 2.1 applied to $u_{0}$ and $\tilde{v}_{0}$ we would obtain

$$
E\left(u_{n}\right)=\left\|u_{n}\right\|^{2}>2 m\left(\frac{m}{c}\right)^{\frac{2}{p-2}}+o(1)
$$

from which the limit value $c$ verifies $c>2^{1-\frac{2}{p}} m$, contrary to our assumption.

Now, we claim that

$$
\tilde{v}_{n} \longrightarrow \tilde{v}_{0} \quad \text { in } H_{0}^{1}(\mathcal{D}) \text {. }
$$


In fact, if this is not the case, we can argue for $\left(\tilde{v}_{n}\right)_{n}$ as we have done for the sequence $\left(u_{n}\right)_{n}$, obtaining that $c \geq 2^{1-\frac{2}{p}} m$, a contradiction. From (3.18), and taking into account (3.17), we infer that (b) holds.

q.e.d.

Remark 3.2 Actually, Proposition 3.1 holds if $c=2^{1-\frac{2}{p}} m$, too. In fact, in such a case, first one proceed as in the proof of Proposition 3.1 and then a further analysis of the Palais-Smale sequence $\left(u_{n}\right)_{n}$ shows that $c=2^{1-\frac{2}{p}} m$ is possible only if the limit function $u_{0}$ is identically zero and $\left(u_{n}\right)_{n}$ tends to split in two different waves, infinitely distant one from the other and such that each one approaches the function $\omega$ on a limit domain that has to be $\mathbb{R}^{N}$. So one can conclude that if $\beta\left(u_{n}\right)=\left(0, \ldots, 0, \zeta_{n}\right)$ then $\zeta_{n} \longrightarrow+\infty$ must hold, by the shape of $\Omega$ in the half space $\left\{x_{N}<0\right\}$.

This means that when $c=2^{1-\frac{2}{p}} m$ a Palais-Smale sequence is composed by two waves "almost minimizing" on strips infinitely distant each other and whose sizes become infinite.

\section{Proof of the main result}

Lemma 4.1 Set

$$
\mathcal{B}:=\inf \left\{E(u): \beta(u)=\frac{Q_{i}^{+}+Q_{i+1}^{-}}{2} \text { or } \beta(u)=-\frac{Q_{i}^{+}+Q_{i+1}^{-}}{2} \text { for some } i \in \mathbb{N}\right\},
$$

it holds

$$
\mathcal{B}>m \text {. }
$$

Proof Let us define

$$
\widetilde{\mathcal{D}}=\left\{x=\left(x^{\prime}, x_{N}\right) \in \mathbb{R}^{N}:\left|x^{\prime}\right|<1\right\} \cup\left\{x=\left(x^{\prime}, x_{N}\right):\left|x_{N}\right|>\frac{1}{2}\right\} .
$$

Observe that $\Omega \subset \widetilde{\mathcal{D}}+\frac{Q_{i}^{+}+Q_{i+1}^{-}}{2}$ and $\Omega \subset \widetilde{\mathcal{D}}-\frac{Q_{i}^{+}+Q_{i+1}^{-}}{2}, \forall i \in \mathbb{N}$, hence, taking into account (2.17), if we prove that

$$
\inf \left\{\|u\|^{2}: u \in H_{0, r}^{1}(\widetilde{\mathcal{D}}),|u|_{p}=1, \beta(u)=0\right\}>m
$$

we are done.

Suppose, by contradiction, that (4.2) does not hold. Then there exists a sequence $\left(u_{n}\right)_{n}$ in $H_{0, r}^{1}(\widetilde{\mathcal{D}}),\left|u_{n}\right|_{p}=1 \forall n \in \mathbb{N}$, such that

$$
\lim _{n \rightarrow+\infty}\left\|u_{n}\right\|^{2}=m
$$




$$
\beta\left(u_{n}\right)=0 \quad \forall n \in \mathbb{N} .
$$

Since the sequence $\left(u_{n}\right)_{n}$ is in $H_{0, r}^{1}(\widetilde{\mathcal{D}})$ and is minimizing for (1.1), then (see [3], for example) there exist a sequence of points $\left(y_{n}\right)_{n}$ in $A$ and a sequence of axially symmetric functions $\left(w_{n}\right)_{n}$ in $H^{1}\left(\mathbb{R}^{N}\right)$ such that

$$
u_{n}(x)=\omega\left(x-y_{n}\right)+w_{n}(x) \quad \text { with } \lim _{n \rightarrow+\infty}\left\|w_{n}\right\|=0 .
$$

From (2.17), (2.18) and the continuity of $\beta$ it follows that

$$
\lim _{n \rightarrow+\infty}\left|\beta\left(u_{n}\right)-y_{n}\right|=\lim _{n \rightarrow+\infty}\left|\beta\left(\omega\left(x-y_{n}\right)+w_{n}(x)\right)-y_{n}\right|=\lim _{n \rightarrow+\infty}\left|\beta\left(\omega(x)+w_{n}\left(x+y_{n}\right)\right)\right|=0 .
$$

By (4.4), (4.6) and (4.5) we have that $u_{n} \rightarrow \omega$ in $H^{1}\left(\mathbb{R}^{N}\right)$; this is not possible, because $u_{n}=0$ on $\mathbb{R}^{N} \backslash \widetilde{\mathcal{D}}, \forall n \in \mathbb{N}$, and $\omega>0$ on $\mathbb{R}^{N}$, so the proof is completed.

q.e.d.

Lemma 4.2 Let $k_{1} \leq k_{2}<\min \left\{\mathcal{B}, 2^{1-\frac{2}{p}} m\right\}$ and assume that

$$
\left\{u \in V: E(u) \in\left[k_{1}, k_{2}\right], \nabla E(u)=0\right\}=\emptyset ;
$$

if $\bar{u} \in E^{k_{2}}$ then there exist $\varepsilon>0$ and a continuous path $\eta:[0,1] \rightarrow E^{k_{2}}$ such that

$$
\eta(0)=\bar{u}, \quad \eta(1) \in E^{k_{1}-\varepsilon}, \quad t \mapsto E(\eta(t)) \text { is decreasing, }
$$

where $E^{b}, b \in \mathbb{R}$, denotes the sublevel

$$
E^{b}=\{u \in V: E(u) \leq b\} .
$$

Proof By assumption (4.7), $\bar{u}$ has a neighborhood $\mathcal{U}(\bar{u})$ in $V$ such that

$$
\|\nabla E(u)\|>\text { const }>0 \quad \forall u \in \mathcal{U}(\bar{u}) .
$$

Then, the Cauchy problem

$$
(C) \quad\left\{\begin{array}{l}
\frac{d}{d t} \Phi(t)=-\frac{\nabla E(\Phi(t))}{\|\nabla E(\Phi(t))\|^{2}} \\
\Phi(0)=\bar{u}
\end{array}\right.
$$

can be locally solved, on $V$. Let $[0, T)$ be the maximal interval where $\Phi$ can be defined; we shall show that $L:=\lim _{t \rightarrow T^{-}} E(\Phi(t))$ is well defined and $L<k_{1}$.

First, we claim that $t \mapsto E(\Phi(t))$ is a decreasing function; indeed, for $t_{1}, t_{2} \in[0, T)$ we have

$$
E\left(\Phi\left(t_{2}\right)\right)-E\left(\Phi\left(t_{1}\right)\right)=\int_{t_{1}}^{t_{2}} \frac{d}{d t} E(\Phi(t)) d t=\int_{t_{1}}^{t_{2}} \nabla E(\Phi(t)) \cdot \frac{d}{d t} \Phi(t) d t=-\left(t_{2}-t_{1}\right) .
$$


From (4.9), and (a) of Proposition 1.1, it follows, in particular, that $T<+\infty$.

Now, let us suppose, by contradiction, that $L \geq k_{1}$ and let $\left(t_{n}\right)_{n}$ be a sequence in $[0, T)$ such that $t_{n} \rightarrow T$ and $\left\|\nabla E\left(\Phi\left(t_{n}\right)\right)\right\| \longrightarrow 0$, as $n \rightarrow+\infty$. Such a sequence $\left(t_{n}\right)_{n}$ must exist, otherwise $\Phi$ would be Lipshitz, because it solves $(C)$, and so we could extend $\Phi$ on an interval $\left[0, T^{\prime}\right)$ with $T^{\prime}>T$, contrary to the maximality of $[0, T)$.

By applying Proposition 3.1 to $\Phi\left(t_{n}\right)$, up to a subsequence, we have two cases:

$\Phi\left(t_{n}\right)$ converges to a function $\bar{v} \in V$; then

$$
E\left(\Phi\left(t_{n}\right)\right) \longrightarrow E(\bar{v})=L \in\left[k_{1}, k_{2}\right], \quad \nabla E\left(\Phi\left(t_{n}\right)\right) \longrightarrow \nabla E(\bar{v})=0,
$$

contrary to (4.7).

$\left|\beta\left(\Phi\left(t_{n}\right)\right)\right| \longrightarrow+\infty$; then, since $\beta$ is continuous, there exists $\bar{t} \in[0, T)$ such that $\beta(\Phi(\bar{t}))=\frac{Q_{i}^{+}+Q_{i+1}^{-}}{2}$ or $\beta(\Phi(\bar{t}))=-\frac{Q_{i}^{+}+Q_{i+1}^{-}}{2}$, for some $i \in \mathbb{N}$, and, moreover, $E(\Phi(\bar{t})) \leq$ $E(\Phi(0))<\mathcal{B}$, by assumption. This is not possible by definition of $\mathcal{B}$ (see (4.1)), so we have the desired conclusion, up to a normalization of the path.

$$
\text { q.e.d. }
$$

Proof of Theorem [1.2 Observe that, taking into account Corollary 2.2, the critical points of $E$ whose critical levels are in $\left(m, 2^{1-\frac{2}{p}} m\right]$ are nonnegative functions, actually positive by the maximum principle.

Then, in order to prove Theorem 1.2, it is sufficient to show that for every $a \in$ $\left(m, 2^{1-\frac{2}{p}} m\right]$ there exists a critical value $c$ for $E$ such $c \in(m, a)$.

Let $S_{k}$ be a "strip" such that $\left\|z_{k}\right\|^{2}<\min \{a, \mathcal{B}\}$ (see (1.4), (2.7) and (2.8)); we claim that $E$ has a critical value $c \in\left(m,\left\|z_{k}\right\|^{2}\right]$. Assume, by contradiction, that no critical value exists in $\left[m,\left\|z_{k}\right\|^{2}\right]$. Then, by Lemma 4.2, there exists a continuous path from $z_{k}\left(x-\frac{Q_{k}^{+}+Q_{k}^{-}}{2}\right)$ to a function $v \in V$ such that $E(v)<m$. But this contradicts (2.15), so we get the claim, by Remark 2.3.

q.e.d.

\section{References}

[1] R.A. Adams - J.J.F. Fournier, Sobolev spaces, 2nd edition, Pure and Applied Mathematics 140, Academic Press - Amsterdam 2003.

[2] A. Bahri - P.L. Lions, On the existence of a positive solution of semilinear elliptic equations in unbounded domains, Ann. Inst. H. Poincaré Anal. Non Linéaire 14 (1997), no. 3, 365-413.

[3] V. Benci - G. Cerami, Positive solutions of some nonlinear elliptic problems in exterior domains, Arch. Rational Mech. Anal. 99 (1987), 283-300. 
[4] V. Benci - G. Cerami - D. Passaseo, On the number of positive solutions of some nonlinear elliptic problems, in Nonlinear Analysis. A tribute in honor of G.Prodi. S.N.S. Pisa (1991), 93-109.

[5] H. Brezis - E. Lieb, A relation between pointwise convergence of functions and convergence of functionals, Proc. A.M.S. 88 (1983), no. 3, 486-490.

[6] G. Cerami - R. Molle - D. Passaseo, Positive solutions of semilinear elliptic problems in unbounded domains with unbounded boundary, Ann. Inst. H. Poincaré Anal. Non Linéaire 24 (2007), no. 1, 41-60.

[7] M.J. Esteban, Nonlinear elliptic problems in strip-like domains: symmetry of positive vortex rings, Nonlinear Anal. 7 (1983), no. 4, 365-379.

[8] M.J. Esteban - P.L. Lions, Existence and nonexistence results for semilinear elliptic problems in unbounded domains, Proc. Roy. Soc. Edinburgh Sect. A 93 (1982/83), no. $1 / 2,1-14$.

[9] J. Molina - R. Molle, On elliptic problems in domains with unbounded boundary, Proc. Edinb. Math. Soc. (2) 49 (2006), no. 3, 709-734..

[10] R. Molle, Semilinear elliptic problems in unbounded domains with unbounded boundary, Asymptotic Analysis 38 (2004), no. 3/4, 293-307.

[11] R. Molle - D. Passaseo, Multiple solutions of nonlinear elliptic Dirichlet problems in exterior domains, Nonlinear Anal. Ser. A: Theory \& Methods 39 (2000), no. 4, 447-462.

[12] R.S. Palais, The principle of symmetric criticality, Comm. Math. Phys. 69 (1979), no. $1,19-30$.

[13] H.C. Wang, Palais-Smale approaches to semilinear elliptic equations in unbounded domains, Electron. J. Differential Equations, Monograph 06, 2004.

[14] J. Wei, On the interior spike solutions for some singular perturbation problems, Proc. Roy. Soc. Edinburgh Sect. A 128 (1998), no. 4, 849-874.

[15] M. Willem, Minimax theorems, Progress in Nonlinear Differential Equations and their Applications, 24. Birkhäuser Boston, Inc., Boston, MA, 1996. 